\title{
THE EFFECT OF IRON DEFICIENCY AND IRON OVERLOAD ON THE EVOLUTION OF CHAGAS DISEASE PRODUCED BY THREE STRAINS OF TRYPANOSOMA CRUZI IN CFW MICE
}

\author{
Maria lúcia Pedrosa,* Marcelo E. Silva, $†$ Márcio E. Silva, $\ddagger$ M. E. C. Silva, $\ddagger$ \\ J. R. Nicoli $\neq$ and E. C. Vieirat \\ *Departamento de Ciéncias Biológicas, Instituto de Ciéncias Exatas e Biológicas, Universidade Federal \\ de Ouro Preto, 35400 Ouro Preto, MG, Brasil. †Departamento de Nutrição, Faculdade de Farmácia, \\ Universidade Federal de Ouro Preto, 35400 Ouro Preto, MG, Brasil. łDepartamento de Bioquímica e \\ Imunologia, Universidade Federal de Minas Gerais, C.P. 2486, 30161 Belo Horizonte, MG, Brasil.
}

(Received 14 February 1990)

\begin{abstract}
CFW mice were fed either on control diet or on iron-deficient diet.
2. After 5 months the mice were infected with $\mathrm{CL}, \mathrm{Y}$ or YuYu strain of Trypanosoma cruzi.

3. On the fifth day after the infection, the mice on control diet were divided in three groups: one group remained as controls, two groups were injected either with desferrioxamine or iron-dextran.

4. The severity of the disease was evaluated by parasitemia and mortality.

5. The experimental groups were compared with the infected group fed on the control diet.

6 . In mice fed on the iron-deficient diet, the disease was more severe for CL strain and less severe for $\mathrm{Y}$ and $\mathrm{YuYu}$ strains.

7. Treatment with desferrioxamine produced a less severe disease with YuYu strain and no difference with the other strains.

8. On treatment with iron-dextran, the disease became more severe with $Y$ and $C L$ strains; no effect was observed with YuYu strain.

9. These findings may be due to intraspecific differences among the strains.
\end{abstract}

\section{INTRODUCTION}

Iron is an essential nutrient for almost all living beings, due to its role in many enzyme systems (Wriggleswort and Baun, 1980). Iron nutriture may affect the host-parasite relationship. If enough iron is not available to a pathogen its propagation may be hampered and, consequently, the infection attenuated (Paine and Finkelstein, 1978). Therefore, when an animal is challenged with an infectious agent, there may be a hypoferremic reaction that would limit the availability of extracellular iron to the parasite (Letendre, 1985). Murray et al. (1978) reported that iron-treated patients were more susceptible to infections by intracellular pathogenic agents. On the other hand, iron is essential for the immune response. Iron deficiency affects both cellular and humoral responses (Galan et al., 1988; Blakley and Hamilton, 1988), which may favor the parasite's survival in the host system. A recent review on the subject of iron status, immune capacity and resistance to infections has shown that there are controversies about the subject (Dhur et al., 1989). The authors remark that, according to the data in the literature, there are two schools of thought that try to explain the role of iron in infection and immunity under the light of the studies stated above and others. On the one hand iron deficiency would predispose to infection due to its deleterious effect on the immune system. On the other hand, iron deficiency would protect against infection, since it would also affect the infectious agent.

In the present work, the effects of the deficiency and supplementation of iron on the evolution of Chagas disease in mice were evaluated. Three strains of Trypanosoma cruzi were studied, namely $\mathrm{CL}, \mathrm{Y}$, and YuYu. It is well known that marked differences exist among these strains of $T$. cruzi (Brener, 1979), so their behavior when faced with iron deficiency was compared.

\section{MATERIALS AND METHODS}

\section{Animals}

Mice of the CFW strain were used. The animals were derived from a breeding nucleus of germfree mice kindly supplied by Dr Morris Pollard, Lobund Laboratory, University of Notre Dame, USA. Part of these animals were conventionalized in our laboratory and have been maintained in a conventional animal room for many generations.

\section{Diet}

Control animals were fed on the semi-purified diet listed in Table 1. Animals fed on the iron-deficient diet received the same diet except that iron was omitted from the formula. The iron-deficient animals were kept in allplastic cages; deionized water was supplied as drinking water from all-glass nipples.

\section{Infection with Trypanosoma cruzi}

Trypanosoma cruzi strains were maintained in our laboratory by passage in CFW mice. The infective forms were 
Table 1. Composition of diets

\begin{tabular}{lc}
\hline Ingredients & g per kg of diet \\
\hline Casein & 150 \\
Soy oil & 50 \\
Starch & 726 \\
Cellulose & 10 \\
Saline mixture* $\dagger$ & 50 \\
Vitamin mixture* & 10 \\
Choline-HCl & 4 \\
$\alpha$-Tocopherol & 0.1 \\
\hline *In iron-deficient diet ferrous sulphate was \\
omitted. \\
+ A.O.A.C. (1980).
\end{tabular}

obtained from infected mouse blood and were injected in experimental animals intraperitoneally. The adjustment of the number of parasites to be inoculated was done by dilution of infected blood with blood from healthy mice. Mice infected with both $C L$ and $Y$ strains were injected with 1400 parasites, whereas the groups infected with the YuYu strain were injected with 15,000 parasites. These inocula were defined as ideal in preliminary experiments.

\section{Parasitemia curve}

Mice were bled daily from the 5 th day of infection for the evaluation of the number of circulating parasites (Brener, 1962). The mice were bled from the tail.

\section{Evaluation of iron deficiency}

From the 2nd day of infection hemoglobin was measured in blood samples collected from each mouse according to Henry et al. (1974). Forty days after infection the animals were sacrificed under ether anesthesia and the blood collected from the axillary plexus into syringes containing heparin. Levels of serum iron were determined (Henry et al., 1974). Livers and spleens were perfused with $0.9 \% \mathrm{NaCl}$, incinerated, and the levels of iron in these organs were determined by the phenanthroline method (A.O.A.C., 1980).

\section{Experimental design}

Pregnant females were fed on iron-deficient diet from the 15 th day of pregnancy. Only the female pups were used in the experiments. The pups were weaned 4 weeks after delivery and fed on iron deficient diet up to 150 days of age. These mice were then inoculated with $T$. cruzi: 12 with the $\mathrm{CL}$ strain, 10 with the $\mathrm{Y}$ strain, and 11 with the $\mathrm{YuYu}$ strain. Ninety female mice of the same age born from mothers fed on the control diet were divided in three groups and inoculated with $\mathrm{CL}, \mathrm{Y}$, or $\mathrm{YuYu}$ strains of $T$. cruzi. At the fifth day of infection each group was divided in three sub-groups: one was injected intraperitoneally with $5 \mathrm{mg}$ of iron-dextran, the second sub-group was injected intraperitoneally with $10 \mathrm{mg}$ of desferrioxamine, and the third group was kept as control. Each sub-group had from 8 to 13 mice. Iron-dextran (ferric hydroxide dextran complex, Sigma Chemical Co., St. Louis, MO) at this dosage is known to cause an overload of iron in mice (Lalonde and Holbein, 1984). Desferrioxamine mesithylate (Desferal, Ciba) is known to cause iron deficiency in mice (Lalonde and Holbein, 1984).

\section{Statistical analysis}

The results were compared by analysis of variance. Comparison of the means was performed by Tucky's test (Levin, 1978).

\section{RESULTS}

\section{$Y$ strain}

The parasitemia and the mortality of mice infected with the $Y$ strain are shown in Fig. 1. The parasitemia was more intense in mice treated with iron-dextran and less intense in mice fed on the iron-deficient diet. These differences were especially obvious on day 10 of infection, when mice treated with iron-dextran had 2.4 times more circulating parasites than the control group, whereas mice fed on the iron deficient diet had 15.6 times less circulating parasites than the control group. The levels of

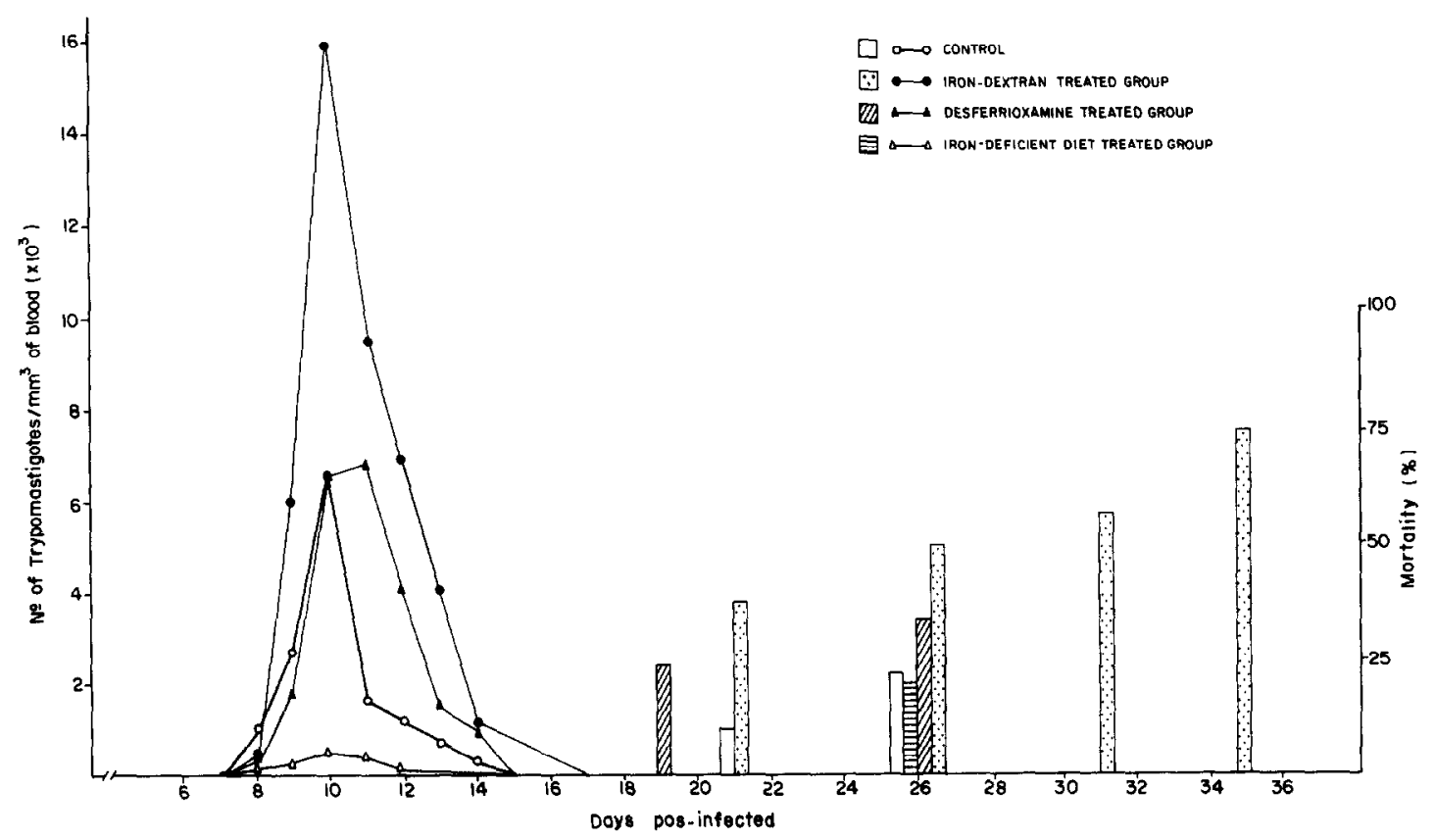

Fig. 1. Parasitemia and cumulative mortality in groups of CFW mice inoculated with $1.4 \times 10^{3}$ blood forms of Trypanosoma cruzi, Y strain. 


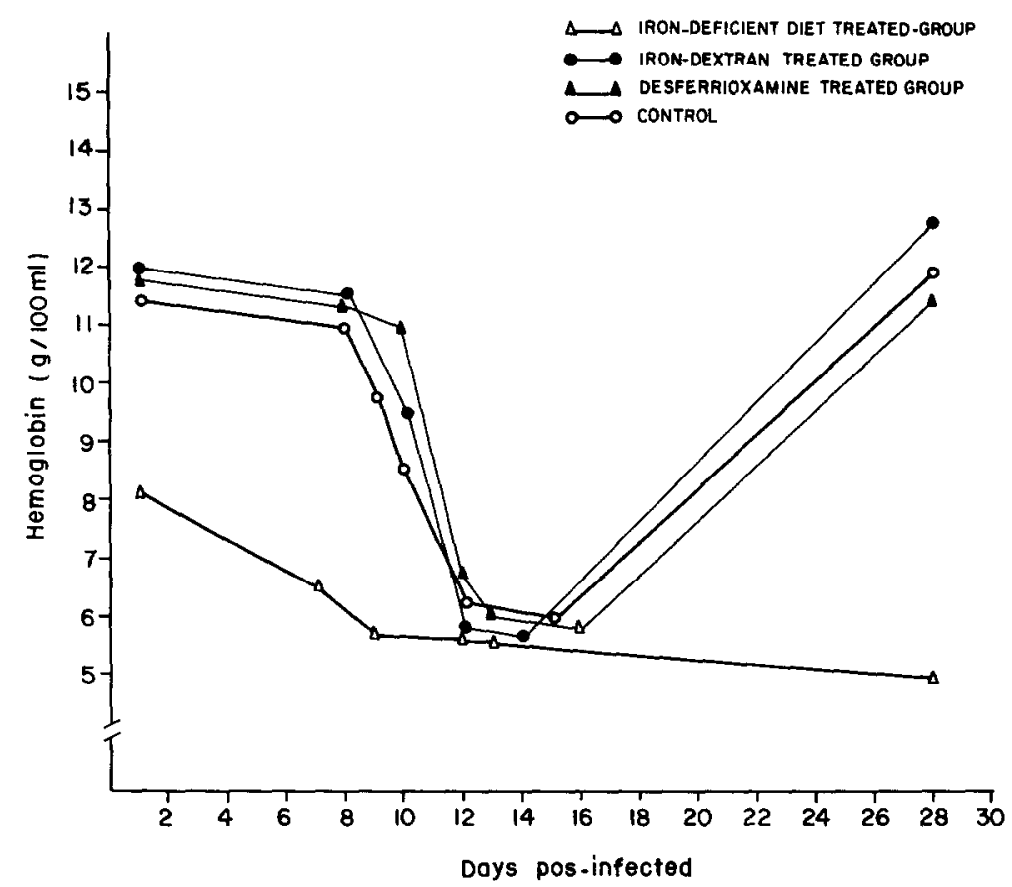

Fig. 2. Hemoglobinemia in CFW mice inoculated with Trypanosoma cruzi, Y strain.

parasitemia in desferrioxamine-treated and control groups were of the same intensity. On the $43 \mathrm{rd}$ day of infection the mortality rates were 75,34 , 22 and $20 \%$ for the iron-dextran-treated, desferrioxamine-treated, control, and iron-deficient groups, respectively.

There was a sharp decrease in the levels of hemoglobin 12 days after infection in control, desferrioxamine-treated, and iron-dextran-treated groups in early infection (Fig. 2). In all these three groups, there was a recovery of hemoglobin levels at the 28th day after infection. Animals fed on iron-deficient diet had levels of hemoglobin 33\% lower than that of other groups before infection. After the infection, there was further decrease of iron levels that maintained itself up to the 28 th day of infection.

\section{CL strain}

The parasitemia and mortality of mice infected with the CL strain of $T$. cruzi are shown in Fig. 3. On day 26 , when a peak in parasitemia occurred, the mean number of parasites in blood of mice fed on iron-deficient diet was 8 times higher than controls. The number of parasites circulating in blood from mice treated with iron-dextran was 3.3 higher than the number found in the control group. Control and desferrioxamine groups showed practically the same pattern of parasitemia. The mortality was higher in animals fed on iron-deficient diet when compared with the other groups (Fig. 3). The levels of hemoglobin after infection are shown in Fig. 4. After the 10th day of infection there was a decrease in the levels of hemoglobin in all groups, this decrease came later in the group fed the iron-deficient diet, after the 20 th day. At the 34th day, the normal levels were recovered except for the mice fed on the iron-deficient diet.

\section{YuYu strain}

Figure 5 depicts the parasitemia and the mortality of the various groups of mice when $\mathrm{YuYu}$ strain was inoculated. The levels of parasitemia on day 26 were highest in the control group and lowest in the desferrioxamine group. Iron-dextran treated mice had lower levels of parasitemia than the control group but higher than the mice fed on the iron-deficient diet. The mortality index followed the same pattern of parasitemia.

The levels of hemoglobin of the various groups infected with the YuYu strain are shown in Fig. 6. The level of hemoglobin in the control group on day 24 of infection was less than half the value obtained before infection. On day 34 after infection the control group recovered the normal values of hemoglobin. Neither the group treated with iron-dextran nor the group treated with desferrioxamine showed any appreciable decrease in the hemoglobin levels. In animals fed on the iron-deficient diet the levels of hemoglobin were low at the beginning of the experiment. There was a further decrease in the course of infection, with no further recovery.

\section{Iron levels in serum, liver, and spleen}

The effect of the different treatments on the levels of iron in serum, liver, and spleen 40 days after infection are shown in Fig. 7. As expected the levels of iron in serum, liver, and spleen were significantly lower in non-infected mice fed on the iron-deficient diet, when compared to their control counterparts fed on the complete diet. On infection with the CL strain of $T$. cruzi the levels of iron in serum of mice fed the control diet were similar to those found on the mice fed the iron deficient diet, and these levels were lower than those found in non-infected mice fed the control diet. The same was true for the other two strains, Y and $\mathrm{YuYu}$. The liver stores of iron were not affected 


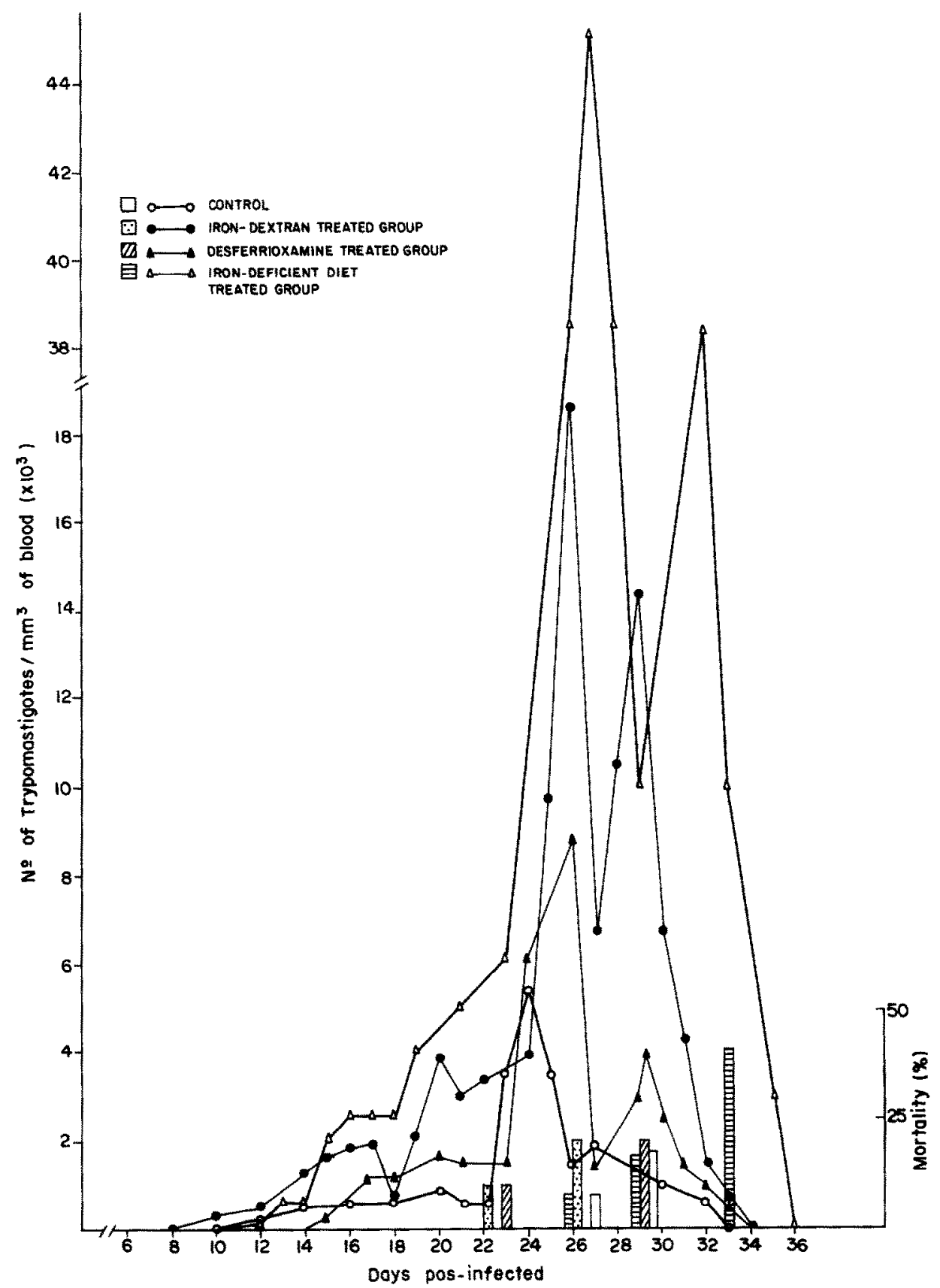

Fig. 3. Parasitemia and cumulative mortality in groups of CFW mice inoculated with $1.4 \times 10^{3}$ blood forms of Trypanosoma cruzi, CL strain.

by infection, when comparing the infected control and non-infected control levels. Mice fed on irondeficient diets consistently showed lower levels of iron in livers than their counterparts fed on the control diets. Levels of iron in spleen were significantly lower in mice infected with the $Y$ strain and fed on the iron-deficient diet when compared with the mice fed on complete diet. However, when the spleen levels of iron in mice infected with the YuYu or the CL strains and fed on complete or iron-deficient diets were compared, it was found that there was no difference between the two groups. Surprisingly, for these two strains, the levels of iron were comparable to the control spleen levels. The levels of iron in mice infected with the YuYu strain and fed on irondeficient diet were lower than control diet levels only in liver.

Treatment with desferrioxamine did not elicit reduction in levels of iron in serum of mice infected with none of the three strains (Fig. 7). It depleted iron from livers of mice infected with $\mathrm{Y}$ and $\mathrm{CL}$ strains, and of spleens of mice infected with the $Y$ strain. Levels of iron in spleens of mice treated with desferrioxamine and infected with the YuYu strain were the same as those of mice infected with the $\mathrm{YuYu}$ strain fed on control diet and not submitted to the 


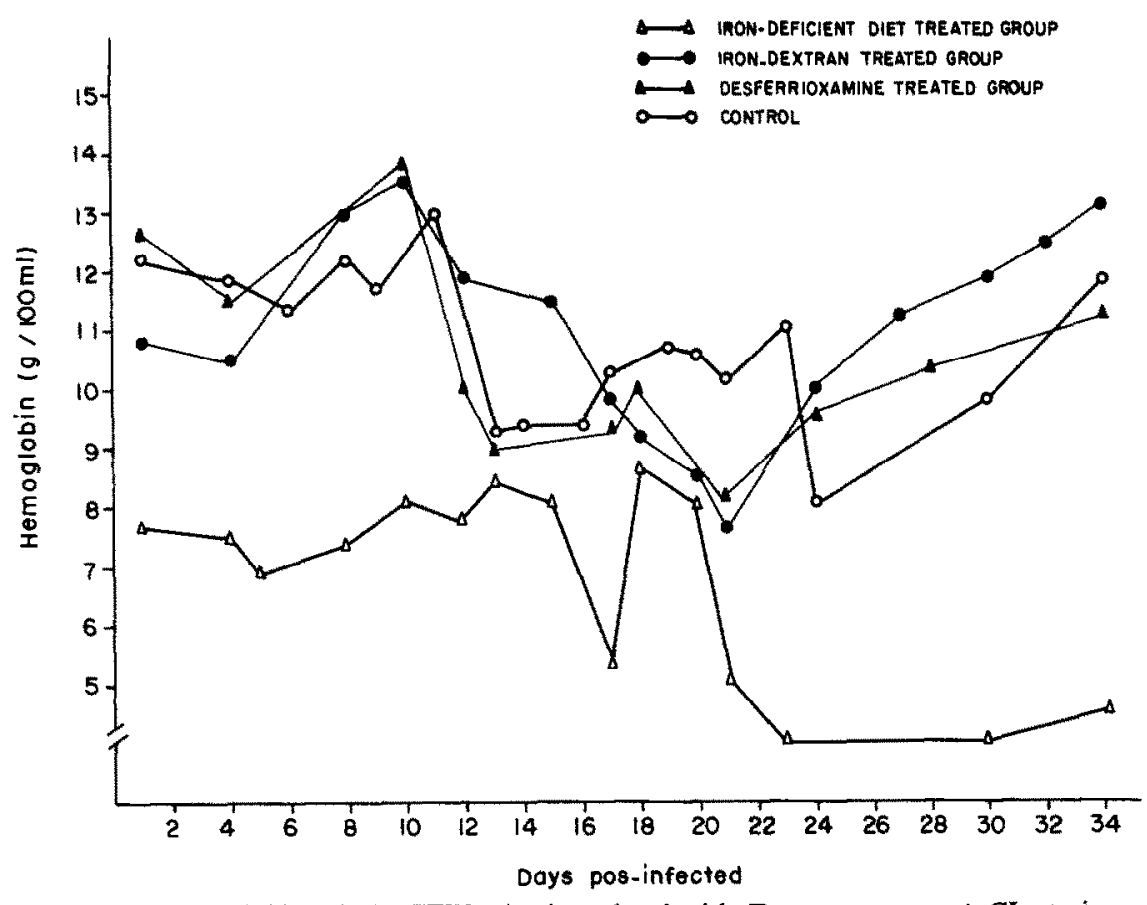

Fig. 4. Hemoglobinemia in CFW mice inoculated with Trypanosoma cruzi, CL strain.

treatment with the iron sequestrant. The same was true for the $\mathrm{CL}$ strain. All these results are in accordance with those found with the iron-deficient diet. The concentrations of iron in livers of mice infected with the YuYu strain and given desferrioxamine, however, were the same found in mice fed on control diet and infected with this strain, and higher than concentrations in infected mice fed on iron-deficient diet.

Treatment with iron-dextran elicited an elevation of iron levels in serum in all infected groups, when these levels are compared to the ones obtained with infected mice fed on the control diet (Fig. 7). Concentrations of iron in livers and spleens were around control levels or above them when irondextran was used, in mice infected with all the three strains.

\section{DISCUSSION}

Iron is required for many enzymatic processes. It has been suggested that its deficiency in the host may affect the reproduction of pathogens (Kochan, 1973). Murray et al. (1978) showed that iron deficiency protected humans against intracellular parasites. The virulence of microorganisms has been attributed, in part, to its efficacy in utilizing iron from the host (Bergeron, 1986). On the other hand, severe iron deficiency may interfere with some host defense mechanisms. There are evidences showing that iron deficiency is reponsible for immunosuppressive effects related to functions of: T-lymphocytes (Blakley and Hamilton, 1988), macrophages (Kuvibidila and Wade, 1987), and neutrophils (Mackler et al., 1984).

When compared with their control counterparts, mice fed on an iron-deficient diet infected with Trypanosoma cruzi showed: (1) lower parasitemia and similar mortality when infected with the $Y$ strain
(Fig. 1); (2) higher parasitemia and higher mortality when infected with CL strain (Fig. 3); (3) lower parasitemia and lower mortality when infected with the YuYu strain (Fig. 5). It is concluded that iron deficiency was beneficial to mice infected with $\mathrm{YuYu}$ strain but malefic to mice infected with the CL strain.

Desferrioxamine, as a chelating agent, has been used in studies on the specific effect of iron on infection, since the immune responses of mice are not affected by injection with this compound (Lalonde and Holbein, 1984). Infection of desferrioxaminetreated mice with the Brazil strain of $T$. cruzi produced a significant reduction in the mortality index (Lalonde and Holbein, 1984). Desferrioxamine also reduced the propagation of amastigotes of $T$. cruzi, in vitro (Loo and Lalonde, 1984). Desferrioxamine did not affect either the parasitemia or the mortality of mice infected with $\mathrm{Y}$ or CL strain of $T$. cruzi when compared with the control groups (Figs 1 and 3). When YuYu strain was inoculated, there was a reduction both in parasitemia and mortality. The results observed may be due to inherent differences in the strains, especially in the multiplication rates. The YuYu strain, in our system, shows peak parasitemia on day 26 in control mice, whereas the $Y$ strain shows peak parasitemia on day 10 of infection and the $C L$ strain on day 24. The inoculum used for the $\mathrm{YuYu}$ strain was higher, but this higher inoculum was unable to anticipate the peak parasitemia. Moreover, the highest numbers of parasites were obtained with the YuYu strain when the peak parasitemia is compared with control mice. So, the YuYu strain takes a longer period of time to reach optimum growth in this mouse system than the other strains, but this longer adaptive period is also followed by an explosion of growth. Therefore, it is possible that due to these characteristics the YuYu strain is more sensitive to iron deficiency than the other strains. This 




Fig. 5. Parasitemia and cumulative mortality in groups of CFW mice inoculated with $1.5 \times 10^{4}$ blood forms of Trypanosoma cruzi, YuYu strain.

was true both with the iron deficient diet and with the desferrioxamine treatment. Desferrioxamine did not affect the growth of the other two strains.

Iron-dextran has been widely used to raise the levels of iron in the body. It has a low toxicity for mice (Holbein et al., 1979). Iron-dextran treatment of mice previously infected with $T$. cruzi, when compared with infected controls, elicited: (1) higher parasitemia and higher mortality when infected with the Y strain (Fig. 1); (2) higher parasitemia and similar mortality when infected with the CL strain (Fig. 3); (3) similar parasitemia and similar mortality with the YuYu strain (Fig. 5). Lalonde and Holbein (1984) reported higher infection rates in iron-dextrantreated mice, previously infected with $T$. cruzi, Brazil strain. This was true in our experiments for $\mathrm{Y}$ and $\mathrm{CL}$ strain. The CL strain was also favoured by the deficiency of iron. For YuYu the increment of iron in the host did not affect its reproduction, but this strain did not tolerate the iron deficiency. For the CL strain the dichotomy of the host-parasite relationship is interesting-in the deficiency of iron, apparently, the immune system of the host is impaired, so the reproduction of the parasite was favoured. In the presence of an excess of iron, the reproduction of the parasite was augmented, and the parasite was again favoured. The YuYu strain had a different behavior; it seems to be sensitive to iron deficiency, may be due to its rather explosive growth. In the excess of iron the reproduction of this strain was not affected. The 


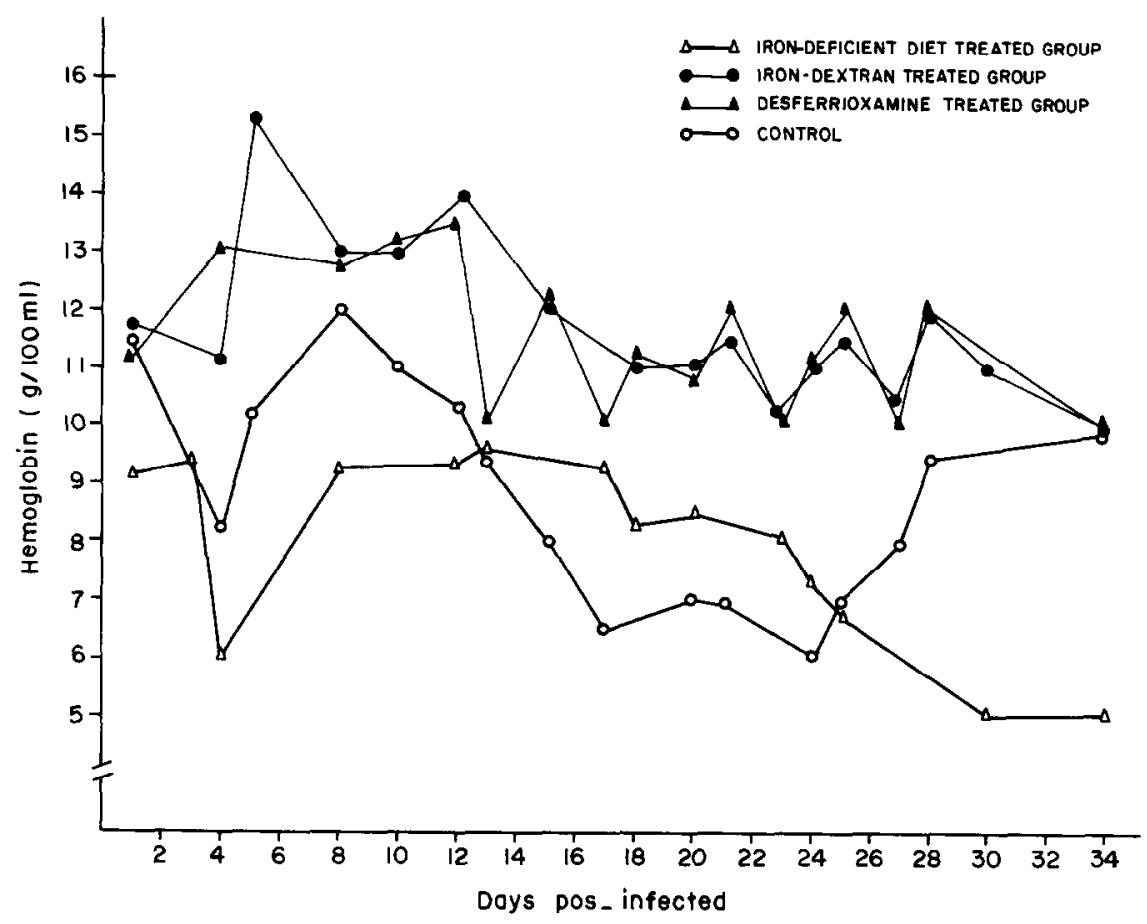

Fig. 6. Hemoglobinemia in CFW mice inoculated with Trypanosoma cruzi, YuYu strain.

Y strain seems to be also sensitive to iron deficiency, and was favoured in excess of iron.

The levels of hemoglobin were sharply reduced in the course of infection with all strains used (Figs 2, 4 and 6). Hematological alterations have been described upon infection with trypanosomes. Anemia was reported in mice infected with $T$. congolense (Esievo et al., 1982) and T. cruzi (Cardoso and Brener, 1980). These alterations have been attributed to different factors: erythrocyte destruction (Esievo et al., 1982), bone marow injury, and hemodilution (Ikede et al., 1977). The present results indicate that the hypohemoglobinemia was transitory except for mice fed on iron-deficient diet. Probably the low iron stores were not sufficient for the compensatory erythropoiesis that follows the anemia. In desferrioxamoxamine-treated animals, however, there was a recovery of hemoglobin levels when infected with the $\mathrm{CL}$ and $\mathrm{Y}$ strains. Anemia was present around the 12th day of infection, following the start of growth of parasites. It is possible that the parasite is using the iron stocks from the host. When the host is on a diet that supplies a sufficient amount of iron it is able to compensate this anemia and recover, but if the host is on a diet that is deficient in iron it is unable to recover and remains anemic.

If the hemoglobin levels show that infection with $T$. cruzi seems to cause a transient anemia from which mice would recover if the iron is available from the diet. This anemia was coincident with the peak of parasitemia in infection with all strains. However, on the 40th day of infection, when mice had already recovered from anemia and were killed, levels of iron in serum were lower than control mice fed a complete diet and not infected (Fig. 7). This could be due to "nutritional immunity", explained by Dhur et al.
(1989) as the capacity of the host to maintain iron unavailable to microorganisms. This way, the host would try to hamper the pathogen growth. However, if the concentration of iron in serum was lower than normal, the iron stocks (liver and spleen) were not affected by infection, (compare the concentrations of iron in livers and spleens of mice fed the control diets with those data of their non-infected counterparts). The stocks of iron in liver were depleted by the iron-deficient diet in all groups studied, and by desferrioxamine in all groups except in the group infected with the YuYu strain. The cause of this different behavior is not known. As for the spleens, the stocks of iron were depleted by the deficient diet and by desferrioxamine in mice infected with the $Y$ strain, which shows tropism towards the spleen (Brener, 1979). The same tendency was observed with the CL strain, although differences were not significant between the control, iron-deficient and desferrioxamine groups. The splenic iron stocks were not depleted by desferrioxamine or iron-deficient diet when mice were infected with the YuYu strain. Preliminary data showed that the YuYu strain was much less infective than the $\mathrm{Y}$ and $\mathrm{CL}$ strains, therefore a higher inoculum was used. So, what was found was that strains with higher infective capacity were able to deplete the splenic stocks, while the lower infectivity strain was not. In the group infected with the $Y$ strain and treated with iron-dextran there was a higher concentration of iron in spleens, which was accompanied by a higher mortality of these mice when compared with controls, which suggests that these higher levels of iron could have favoured the parasite rather than the host.

Intraspecies variation of Trypanosoma cruzi have been previously described. Filardi and Brener (1987) have determined the susceptibility of 47 strains to two 

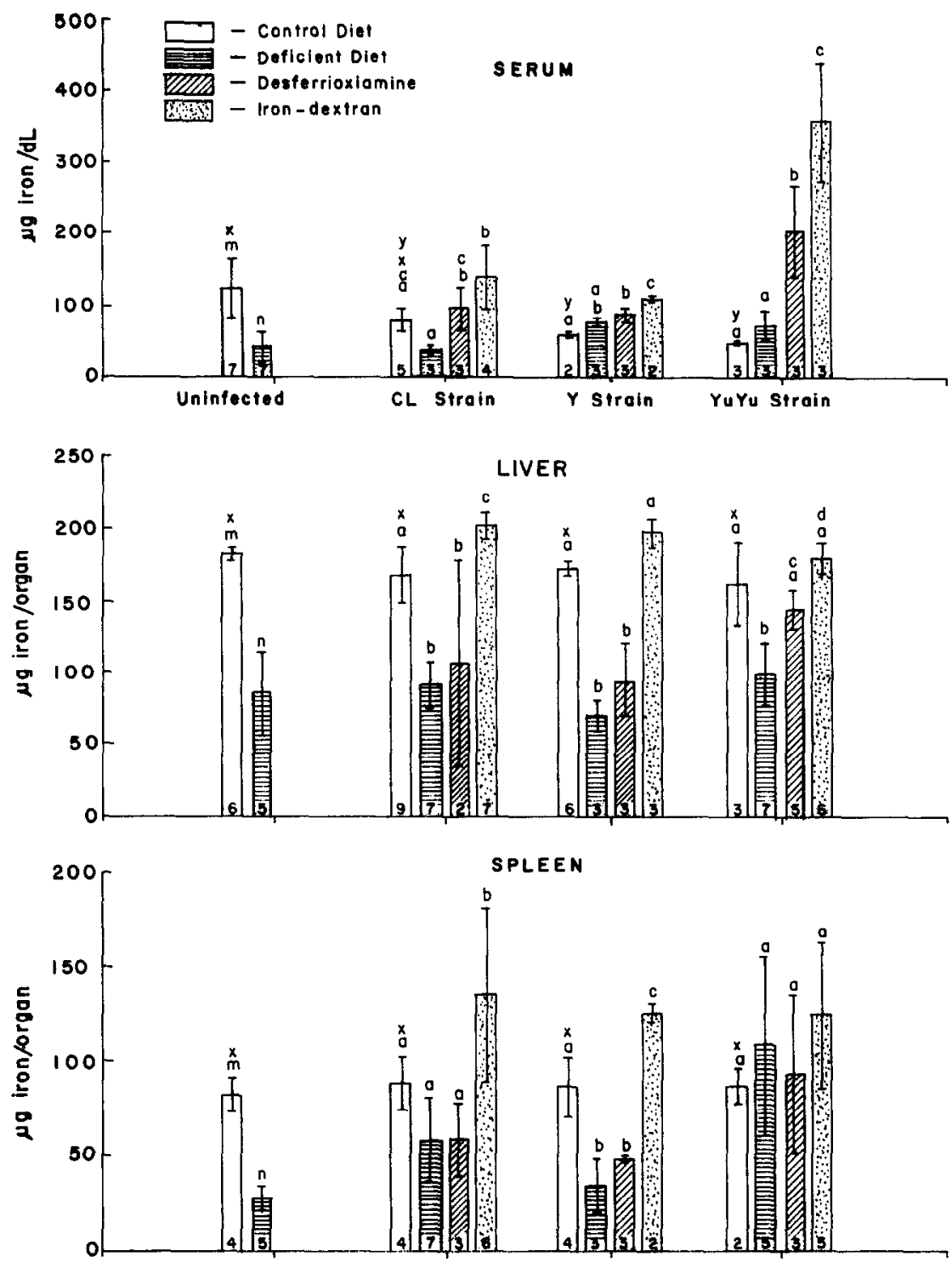

Fig. 7. Iron levels in serum, liver, and spleen of mice infected with either CL, Y or YuYu strains of Trypanosoma cruzi, fed either control or iron-deficient diets, treated or not with iron-dextran or desferrioxamine, killed $\mathbf{4 0}$ days after infection. Data obtained from non-infected mice fed the two different diets are also shown. Bars are the means of concentrations of iron in the different compartments, vertical lines represent the standard deviations of the means. The number of mice used for each assay are between parenthesis, different letters represent statistical difference $(P<0.05)$ in each compartment. Comparisons were made: (1) within groups infected with each strain and bearing different iron status (a, b, c, d); (2) between non-infected mice fed on the control and iron-deficient diets $(m, n) ;(3)$ between infected and non-infected mice fed on the control diet $(x, y)$.

drugs that have been used as a treatment to Chagas disease, benzidazole and nitrofurtimox. The efficacy of these drugs varied from 0 to $100 \%$ among the several strains of $T$. cruzi. For the strains we have studied, $\mathrm{CL}, \mathrm{Y}$ and $\mathrm{YuYu}$ the percentage cure was 100,47 and $0 \%$, respectively, which suggests that these strains indeed differ from each other in their response to environmental stress. Other variations among $T$. cruzi strains have been related with the morphology of the parasite, intracellular distribution, etc. (Brener, 1977; Tafuri, 1987). The results herein show that decreased and increased levels of body iron affect the course of Chagas disease in mice in different manners, depend- ing on the strain that is inoculated. These differences may be considered as the expression of intraspecific variations.

Acknowledgements - This work was supported by Conselho Nacional de Desenvolvimento Científico e Tecnológico (CNPq), Financiadora de Estudos e Projetos (FINEP) and Fundação de Amparo à Pesquisa do Estado de Minas Gerais (FAPEMIG). We are grateful to Dr Leda Q. Vieira for suggestions and careful review of the manuscript and to Dr Roldão Roosevelt Urzedo de Queiroz for technical help. 


\section{REFERENCES}

A.O.A.C. (1980) Official Methods of Analysis of the Association of Official Analytical Chemists. 3rd edn; Washington, D.C.

Bergeron R. J. (1986) Iron: a controlling nutrient in proliferative processes. Trends Biochem. Sci. 13, 133-136.

Blakley B. R. and Hamilton D. L. (1988) The effect of iron deficiency on the immune response in mice. Drug Nutrient Interactions 5, 249-255.

Brener Z. (1962) Therapeutic activity and criterion of cure un mice experintentally infected with Trypunosoma cruzi. Rev. Inst. Med. Trop. São Paulo 4, 389-396.

Brener Z. (1977) Intraspecific variations in Trypanosoma cruzi: two types of parasite populations presenting distinct characteristics. In Chagas' Disease. Scientific Publication No. 347, pp. 11-21, Pan American Health Organization, New York.

Brener Z. (1979) O parasito: Relações Hospedeiro-parasito. In Trypanosoma cruzi e Doença de Chagas, (Edited by Brener Z. and Andrade Z.) pp. 1-44. Editora Guanabara Koogan, Rio de Janeiro.

Cardoso J. E. and Brener Z. (1980) Hematological changes in mice experimentally infected with Trypanosoma cruzi. Mem. Inst. Oswaldo Cruz 75, 97-104.

Dhur A., Galan P. and Hercberg S. (1989) Iron status, immune capacity and resistance to infections. Comp. Biochem. Physiol. 94A, 11-19.

Esievo K. A., Saror D. I., Ilemobade A. A. and Hallaway M. H. (1982) Variation in erythrocyte and free sialic acid serum concentrations during experimental Trypanosoma vivax infection in cattle. Res. Vet. Sci. 32, 1-5.

Filardi, L. S. and Brener Z. (1987) Susceptibility and natural resistance of Trypanosoma cruzi strains to drugs used clinically in Chagas disease. Trans. Royal Soc. Trop. Med. Hyg. 81, 755-759.

Galan P., Davila M., Mekki N. and Hercberg S. (1988) Iron deficiency, inflammatory processes and humoral immunity in children. Int. J. Vit. Nutr. Res. 58, 225-230.

Henry R. J., Cannon D. C. and Winkelman J. W. (1974) Clinical Chemistry: Principles and Technics, 2nd edn., Harper and Row, New York.
Holbein B. E., Jericho K. W. F. and Likes G. C. (1979) Neisseria meningitidis infection in mice: influence of iron, variations in virulence among strain, and pathology. Infect. Immun. 24, 545-551.

Ikede B. O., Lule M. and Terry R. J. (1977) Anaemia in trypanosomiasis: mechanisms of erythrocyte destruction in mice infected with Trypanosoma congolense or T. brucei. Acta Trop. 34, 53-60.

Kochan, I. (1973) The role of iron in bacterial infections with special consideration of host-turbecule bacillus interaction. Current Top. Microbiol. Immunol. 60, 1-30.

Kuvibidila S. and Wade S. (1987) Macrophage function as studied by the clearance of ${ }^{125}$ I-labeled polyvinylpyrrolidone in iron-deficient and iron-replete mice. J. Nutr. 117, $170-176$.

Lalonde R. G. and Holbein B. E. (1984) Role of iron in Trypanosoma cruzi infection of mice. J. Clin. Invest. 73, $470-476$.

Levin J. (1978) Estatística Aplicada a Ciências Humanas, 2nd edn., pp. 174-190, Copyright 1978 Editora Harper e Row do Brasil Ltda, Rio de Janeiro.

Letendre E. D. (1985) The importance of iron in the pathogenesis of infection and neoplasia. Trends Biochem. Sci. 12, 166-168.

Loo V. G. and Lalonde R. G. (1984) Role of iron in intracellular growth of Trypanosoma cruzi. Infect. Immun. 45, 726-730.

Mackler B., Person R., Ochs H. and Finch C. A. (1984) Iron deficiency in the rat. Effects on neutrophil activation and metabolism. Pediat. Res. 18, 549-551.

Murray M. J., Murray A. B., Murray M. B. and Murray C. J. (1978) The adverse effect of iron repletion on the course of certain infections. Brit. Med. J. 2 , 1113-1115.

Paine S. M. and Finkelstein R. A. (1978) The critical role of iron in host-bacterial interactions. J. Clin. Invest. 61, $1428-1440$.

Tafuri W. L. (1987) Patogenia da doença de Chagas. Rev. Inst. Med. Trop. São Paulo 29, 194-199.

Wriggleswort J. M. and Baun H. (1980) The biochemical functions of iron. In: Iron in Biochemistry and Medicine, (Edited by Jacobs A. and Worwood M.) Vol. II, pp. 29-86. Academic Press, New York. 